\title{
Obesity, Risk Factors and its Pharmocological Treatments
}

\author{
Mahendran C. ${ }^{1}$, Midhun Kumar G.H. ${ }^{2}$, Parimala K. ${ }^{3}$, Shanmuga Priyan ${ }^{4}$ \\ ${ }^{1}$ Assistant Professor, Department of Community Medicine, Meenakshi Medical College Hospital and Research \\ Institute, Enathur, Kanchipuram, Tamilnadu, India, ${ }^{2}$ Associate Professor, Department of Community Medicine, \\ Meenakshi Medical College Hospital and Research Institute, Enathur, Kanchipuram, Tamilnadu, India, ${ }^{3}$ Professor \\ \& HOD, Department of Pharmacology, Meenakshi Medical College Hospital and Research Institute, Enathur, \\ Kanchipuram, Tamilnadu, India, ${ }^{2}$ Associate Professor, Department of Pharmacology, Meenakshi Medical College \\ Hospital and Research Institute, Enathur, Kanchipuram, Tamilnadu, India
}

\begin{abstract}
In recent years, Obesity has become one of the major health issue affected the children to adult people. Changes in life style, food habits and stress management has been the disease related problems not only for obesity for many health complications. Many of the drugs has been developed in the market to the availability of people. Some of the drugs produced by the pharmaceutical companies shows side effects after using the medicines. The most recently used drugs are Phentermine, Diethylpropion, Phendimetrazine, Benzphetamine, and Mazindol are referred for the obese patients for short term treatment. Sibutraminea,the sympathomimetic are referred as long term medication for the patients with obesity. Many of the drugs are banned from the pharmaceutical industry due to the adverse effects it has on patients health. This paper discusses the major reasons and issues related with obesity. The medications and treatments in available recent times are also discussed.
\end{abstract}

Keywords: Obesity, risk factors, treatment, BMI, Gynaecological Problems.

\section{Introduction}

The world Health Organization has reported this obese issue as "excessive and abnormal fat accumulates develops risks to health" it is usually calculated with the formula of BMI known as Body Mass Index it presents the level of fat accumulated in the body ${ }^{[1]}$. The available treatment options are set according to the range of body mass index, circumstances and other health problemsare taken into record to avoid any future health complications. However for endocrinologists are trying to resolve the major health consequence of developing obesity and type 2 diabetic patients ${ }^{[2]}$. Research study on

\section{Corresponding Author:}

\section{Mahendran C.}

Assistant Professor, Department of Community Medicine, Meenakshi Medical College Hospital and Research Institute, Enathur, Kanchipuram, Tamilnadu, India

e-mail: mahendranc@mmchri.ac.in obese reports that around $30 \%$ of middle age european people are obese and in united states nearly around 3 lakhs people died every year those are obese. Obesity patients are tend to associate with the risk of developing higher mortality rate. The average obese body weight range patients are saved without any risk development. Life style changes, junk food eaters and lack of exercise among the people are the biggest causes of obese people. There are certain technologies available for the people such as anthropometric measurement namely skin-fold thickness, measurement of waist and hip range are used to assess the complications in obese patients associated with type 2 diabetes mellitus also cardiovascular diseases. In 2015-16 THE WHO has announced obesity as epidemic and this has arised worldwide about 1 billion and 600 million people are adults with obese. Public health sector has announced many health insurance schemes for aged people to recover their health ${ }^{[3]}$. Obese are also tend to develop gastroenterology disorders such as hernia, colon cancer, haemorrhoids so on ${ }^{[4]}$.

Obesity; Risk Factors: The public are not aware of such health complications associated with obesity, 
and how it influences peoples life in the way of affecting health and economical imbalance in running families these are all the important problems related with the disease. It arise when the energy imbalance in energy utilized and spent in day to day life when the calories are not utilized properly it is converted into fat and gets deposited in the body. The major reasons for change in people behaviour towards health are the availability of nutrition and the less nutritious foods the industries growth all these problems affects the people health directly and ends up in obesity.

Gynaecological Problems: Obese women health definitely varies from normal health women. Starting from menstrual issues irregulations and amenorrhoea that is long time absence of menstrual cycle. The menstrual issues in women arises because of the hormonal imbalance in the female body. This change is due to the upregulation of androgens and oestrogen levelsand increased conversion of androgen to estrogen in female obese patients. Research studies also suggests that the obese women are more prone to endometrial cancer with the highest production rate of oestrogen in the endometrium Types of cancers arise in obese are breast cancer gall bladder cancer and ovarian cancer. $70 \%$ of the obese women are developing polycystic ovarian disease or syndrome commonly known as PCOD or PCOS. During pregnancy this overweight issue leads to hypertension, diabetes and pre-eclampsia and big headed baby development are seen ${ }^{[5]}$.

Pulmunory Functioning Issues: Research reports says that the obesity problem is related with sleeping disorders known as (OSA) OBSTRUCTIVE SLEEP APNEA. The enlargement of neck width range is associated with sleep disorder in obese patients. There are two major reasons to affect health because of the tissue fat has been attached in lumen and secondly the fat tissues blocks the airways and collapses the ranges of air passes through the way. Breathing issue asthma is critical disease even that may develop in the obese patients. There are also evidences provided to prove obese patients are developing asthmatic issues. Seventy $\%$ of the patients with asthma are diagnosed from the obese patients. Active mechanism is obese people are related with asthma complaint because of the hyperresponsive airways and decrease in function. The block of inhibitors results in the hyper reaction where the inhibited agents are cytokines, chemokines and adipocyte factor leptin ${ }^{[6]}$.
Coronary Heart Disease: Obesity majorly affects the heart by blocking pathways by fat accumulation and increases the chances of getting cardiovascular disorder. The metabolic rate of obese patients compared with normal healthy patients are low in range leads to many diseases and disorders many heart disease. Hypertension reporting studies reveals that the adipose tissue containing fat are accumulated in high level is indicated by calculating the BMI (Body Mass Index). The blood circulation is reduced and pumping capacity of blood to the heart is reduced by the deposited fat in the arteries leads to severe hart blocks and heart attacks. Nearly millions of people die every year because of obesity related heart problems and attacks. Overweight and lacking in exercise and the fried food items are major reasons for obesity leads to cardiovascular diseases. Daily walking for $40 \mathrm{mins}$ can eventually reduce such coronary diseases by burning the excess calories we can reduce the fat accumulation in body ${ }^{[7]}$.

Anti-Obesity Medicines: Many number of drugs are prescribed for obese people for weight reduction. These recent medicines are approved during past years. Some of the pharmaceutical companies still developing medicines for the obesity patients. Drug called topiramate anti-epileptic medicine used for migraine and roflumilast plays role in pulmonary disorders. Bupropion are known to treat depression. Other important drugs for the reducing weight is not the first line treatment but adopted for the obesity treatment ${ }^{[8]}$. In developed countries phentermine and the drug diethylpropion are distributed largely in 1970. These releases medicinal compounds such as dexfenfluramine and fenfluramine. It is known that these agents had potential to develop hypertension of pulmonary and it was highly put under risk order which works against the weight loss process ${ }^{[8]}$.

Diethylpropion: It is a compound consists of phenylalanine ring compound with small sympathomimetic property and present with stimulants shows effect thanamphetamine. Diethyl had approved by Food drug and administration for obesity treatment in 1959. It has been in use for weight reduction and the studies had been under evaluation for the use of this medicine for long term. Analysis called meta analyses assess the action of this diethylpropion drug for in different individuals and presented in the published report between 1960\& 1980. These studies were carried out for at least more than 20 weeks. The obese patients are treated with these drug to ensure weight loss each 
individual has at least lost three kilo of weight without any side effects ${ }^{[9]}$.

Phentermine: It is sympathomimec anorectic medicine belongs to the phenylethelamine class pharmacologically relevant to the amphetamine. The action of this medicine is to work in the stimulation of hypothalamus in the adrenal glands releasing the norepinephrine reducing the abdominal fat ${ }^{[10]}$. To notice it is not established as a first line medicine such as amphetamine in treating the obesity by weight loss. Central nervous system activity and metabolic activity may be involved.FDA has approved this medicine to treat obesity for week based treatment named monotherapy because of this single medicine for weight reduction, physical exercise and calorie reduction in obese patients with highest BMI rate. TILL 2006 this medicine was prescribes as an anti-obese medicine in the U.S country. Some of these formulations had side effects also such as headache, increased heart rate and constipation ${ }^{[11]}$.

Cathin: It is one of the anti-diabetic drug derived from the formulations of amphetamine drugs in the pharmaceutical industry for the past years of treatment for obesity patients. It is an alkaloid derived from the leaf of khat plant it is licensed to use in the short period of time for diet improper obesity. Pharmacology formulations of this plant works as an amphetamine drug utilizes which induce the anorexia and sensory simulating, urine retention associated disease. Variation in medicine dosage can increase heart rate and many safety related problems are considered while giving the medicine. Monotherapy of cathin can be prescribed for atleast 20 weeks and reported safe. The use of this medicine is not advised for long time and still cannot be identified even in many clinical trials specially with cardiovascular diseases ${ }^{[12]}$.

Pharmacology is an major part in treating the obesity disease its role in weight loss is uncountable. Considering the major health issue and death rate are the complications taken into consideration by the industries to produce drugs that are available for people with obesity. Taking drugs can reduce the health ailments but real weight loss procedures should be followed by the patient to ensure healthy body The fact is the use of medicines for obesity will work only while you are using the medicines later it wont show its action. So use of medications is only a temporary path and having a healthy life still with exercise and taking nutritious food are the key to success ${ }^{[13]}$.
Weight Management: A group of obese patients were analysed for weight loss in research it was separated into 3 groups and after period of six months patients from three different groups were put into weight loss pattern they all showed average range of change in their weight. The average weight loss were seen in different groups. The analysis were brought to secondary stage the patients with obesity are put into these group with mixed number of people from all the groups there was change in weight loss pattern. These patterns are finalized and revealed that these low-energy intake of diet shows the effects in weight loss treatment. Hence it is proved that weight loss is possible with proper diet intake. Reduced calories will burn fat and also stops the accumulation of fat in body of both male and female ${ }^{[14]}$.

Food Systemic Approach Preventing Obesity: Attention seeking towards current policies and system for food may create an impact towards public health and things related to diet may reduce the diseases in chronic level. An organizational authority of food system says that the food should be available for all the people that is availability of food, and accessibility of food and affordability to all the people. Reduction in healthy food supply induces the malnutrition in children it also creates many deficiencies in human health. There are efforts taken to reduce the space between the science people and public policy related issues that requires changes in the improvement of food availability and healthy living of people in the risky population ${ }^{[15]}$.

Dietary Therapy: Obesity patients can receive charts and patterns for the diet intake from nutritionists according to their weight loss goals and risks in their health. The obese should follow the medications properly for long time and some of the changes should take place in their life style diet intake for the patients. To follow these procedures patients should participate in the health programs conducted by the health institutes. Normally fiber, grains and fruit juice consumption plays a major role in weight reduction. Fruits intake $30 \%$ in daily diet can reduce $70 \%$ of weight in obese patients recommended by nutritionists. Weight loss process with some nutritious food for a long period of time helps in weight loss also for healthy living of people ${ }^{[16]}$.

\section{Conclusion}

People have awareness towards a healthy lifestyle and exercises are less nowadays. That should be managed and maintained by people for make a disease free life. 
So many health complications are followed in the absence of healthy diet such as cardiovascular diseases, neurological disorders, memory issues, menstrual problems in women and also developing cancers in many obesity patients. All these can be avoided by having a healthy diet and following exercises are key to have healthy life. Alcohol consumption is also one of the major health complications related to obesity. To avoid these complications and to maintain a healthy life style these implications should be followed throughout the weight loss process to lead a disease free healthy lifestyle.

\section{Ethical Clearance: Nil}

Source of Funding: Meenakshi Academy of Higher Education and Research, Chennai, India

Conflict of Interest: Nil

\section{References}

1. Glandt M, Raz I. Present and future: pharmacologic treatment of obesity. Journal of obesity. $2011 \mathrm{Feb}$ $8 ; 2011$.

2. Ruban A, Stoenchev K, Ashrafian H, Teare J. Current treatments for obesity. Clinical Medicine. 2019 May;19(3):205.

3. Wyatt HR. Update on treatment strategies for obesity. The Journal of Clinical Endocrinology \& Metabolism. 2013 Apr 1;98(4):1299-306.

4. Nammi S, Koka S, Chinnala KM, Boini KM. Obesity: an overview on its current perspectives and treatment options. Nutrition journal. 2004 Dec;3(1):1-8.

5. Ogunbode AM, Fatiregun AA, Ogunbode OO. Health risks of obesity. Annals of Ibadan postgraduate medicine. 2009;7(2):22-5.

6. Beccuti G, Pannain S. Sleep and obesity. Current opinion in clinical nutrition and metabolic care. $2011 \mathrm{Jul} ; 14(4): 402$.
7. Lavie CJ, McAuley PA, Church TS, Milani RV, Blair SN. Obesity and cardiovascular diseases: implications regarding fitness, fatness, and severity in the obesity paradox. Journal of the American College of Cardiology. 2014 Apr 15;63(14):134554.

8. May M, Schindler C, Engeli S. Modern pharmacological treatment of obese patients. Therapeutic Advances in Endocrinology and Metabolism. 2020 Jan;11:2042018819897527.

9. Rodgers RJ, Tschöp MH, Wilding JP. Anti-obesity drugs: past, present and future. Disease models \& mechanisms. 2012 Sep 1;5(5):621-6.

10. Kang JG, Park CY. Anti-obesity drugs: a review about their effects and safety. Diabetes \& metabolism journal. 2012 Feb 1;36(1):13-25.

11. Martins A, Morgado S, Morgado M. Anti-obesity drugs currently used and new compounds in clinical development. World Journal of Meta-Analysis. 2014 Nov 26;2(4):135-53.

12. May M, Schindler C, Engeli S. Modern pharmacological treatment of obese patients. Therapeutic Advances in Endocrinology and Metabolism. 2020 Jan;11:2042018819897527.

13. Coelho RC. Anti-obesity drugs: a necessary part of treatment. J Obes Weight-Loss Medic. 2015;1:001e..

14. Smethers AD, Rolls BJ. Dietary management of obesity: cornerstones of healthy eating patterns. Medical Clinics. 2018 Jan 1;102(1):107-24.

15. Finney Rutten L, Yaroch AL, Patrick H, Story M. Obesity prevention and national food security: a food systems approach. International Scholarly Research Notices. 2012;2012.

16. Wirth A, Wabitsch M, Hauner H. The prevention and treatment of obesity. Deutsches Ärzteblatt International. 2014 Oct;111(42):705. 